\title{
Göttinger Vorgehen bei der Aufarbeitung von Sentinel-Lymphknoten beim malignen Melanom
}

\section{Our Approach in Goettingen to Pathology of Sentinel Lymph Nodes for Melanoma}

Autoren

Institut
H. P. Bertsch, C. Mitteldorf, L. Kretschmer

Abteilung für Dermatologie und Venerologie, Georg-August Universität Göttingen

\section{Bibliografie}

Dol $10.1055 / \mathrm{s}-0028-1119443$

Akt Dermatol 2009; 35:

84-86 ๑ Georg Thieme

Verlag KG Stuttgart · New York ISSN 0340-2541

\section{Korrespondenzadresse}

Dr. med. Hans Peter Bertsch

Abteilung für Dermatologie und Venerologie

Georg-August Universität

Göttingen

Von-Siebold-Straße 3

37075 Göttingen

derhigoe@med.uni-

goettingen.de

\section{Zusammenfassung \\ $\nabla$}

Die Aufarbeitung von Sentinel-Lymphknoten beim malignen Melanom beinhaltet einen Kompromiss zwischen Effizienz und Arbeitsaufwand, der sich in unterschiedlichen Vorgehensweisen bzw. Protokollen wiederspiegelt. Die Herausfor-

\section{Einleitung \\ $\nabla$}

Die Sentinel-Lymphonodektomie (SLNE) gilt mittlerweile als Standard-Untersuchung beim primären malignen Melanom. Die Indikation zur SLNE wird meist ab einer Tumoreindringtiefe (Breslow-Index) des Primärtumors von $>1 \mathrm{~mm}$ gestellt, beim Vorliegen weiterer Risikofaktoren wie Clark IV, Ulzeration oder vielen Mitosen kann die SLNE auch schon ab einer Tumordicke von 0,76 mm erwogen werden. Der pathologische Status der Sentinel Lymphknoten (SLKs) wurde in vielen Studien als wichtigster prognostischer Faktor für das Überleben von Patienten der klinischen Stadien I und II etabliert [2-4]. Bei $15-34 \%$ der Patienten in diesem Stadium sind Metastasen im SLK nachweisbar [1-8]. Die Detektionsraten sind deshalb so heterogen, weil sie zum einen durch die Prognosefaktoren des Primärmelanoms (Breslow, Ulzeration), zum anderen aber auch durch die Art und Weise der histopathologischen Aufarbeitung beeinflusst werden.

Die ideale pathologische Aufarbeitung von SLKs sollte darauf ausgelegt sein, so viele Metastasen wie möglich zu finden. Der Anspruch einer kompletten Aufarbeitung eines SLNs erweist sich in der täglichen Praxis jedoch als nahezu unmöglich. Eine komplette Aufarbeitung würde beispielsweise für $1 \mathrm{~cm}$ Lymphknoten(LK)-Gewebe mehr als 3000 Serienschnitte erfordern, was einen unermesslichen Arbeitsaufwand und hohe Kosten nach sich ziehen würde. Verschiedene derung für den Befunder besteht in der Detektion von Mikrometastasen und Einzelzellen durch Verwendung mehrerer immunhistochemischer Antikörper. Die prognostische Bedeutung nodaler Metastasierungsmuster und minimaler Tumorlast wird zukünftigen multizentrischer Studien mit Langzeitverläufen überlassen bleiben.

publizierte Protokolle der histopathologischen Aufarbeitung von SLKs versuchen daher einen Kompromiss zwischen einer möglichst hohen Effizienz beim Auffinden der (Mikro-)Metastasen und einem vertretbaren Arbeitsaufwand für das pathologische Labor herzustellen.

\section{Verfahren zur Aufarbeitung des SLKs $\nabla$}

Das Erkennen von mit bloßem Auge sichtbaren Metastasierungen im SLK dürfte kein Problem darstellen. Andererseits ist der Begriff der „Mikrometastase“ beim Melanom nicht genau definiert. Kleine Gruppen von wenigen Melanomzellen, die bereits Ausdruck einer intranodalen Proliferation sein könnten, lassen sich jedoch nur bei subtiler Durchmusterung möglichst des gesamten SLKs finden. Mehrere Vorgehensweisen sind in der Literatur beschrieben und werden nachfolgend aufgeführt.

Nach einer Hypothese von Cochran et al. [9] findet sich die Mehrzahl der Mikrometastasen im SLK in dessen zentralen Ebene, also an den Schnittebenen eines entlang der Längsachse durch den Hilus mittig halbierten SLKs. Die Anatomie eines mittig durch seine größte Zirkumferenz geteilten LK ist in $\bullet$ Abb. 1 schematisch dargestellt. Cochran schlug die Durchführung von je 10 Serienschnitten ausgehend von beiden Oberflächen des mittig geteilten Lymphknotens vor. Dabei kamen H\&E sowie der immunhistochemischen Marker S100 und HMB45 zur An- 


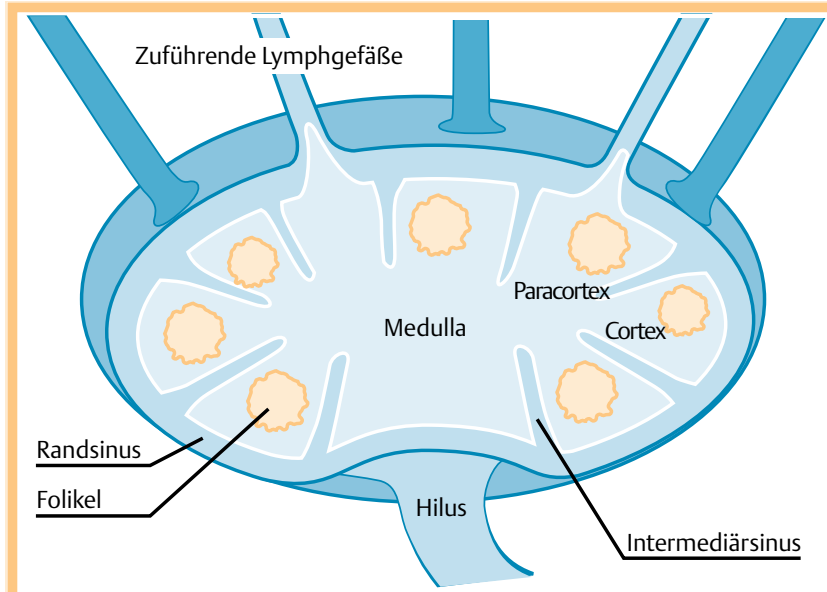

Abb. 1 Schematische Darstellung des anatomischen Aufbaus eines entlang der Längsachse halbierten Lymphknotens.

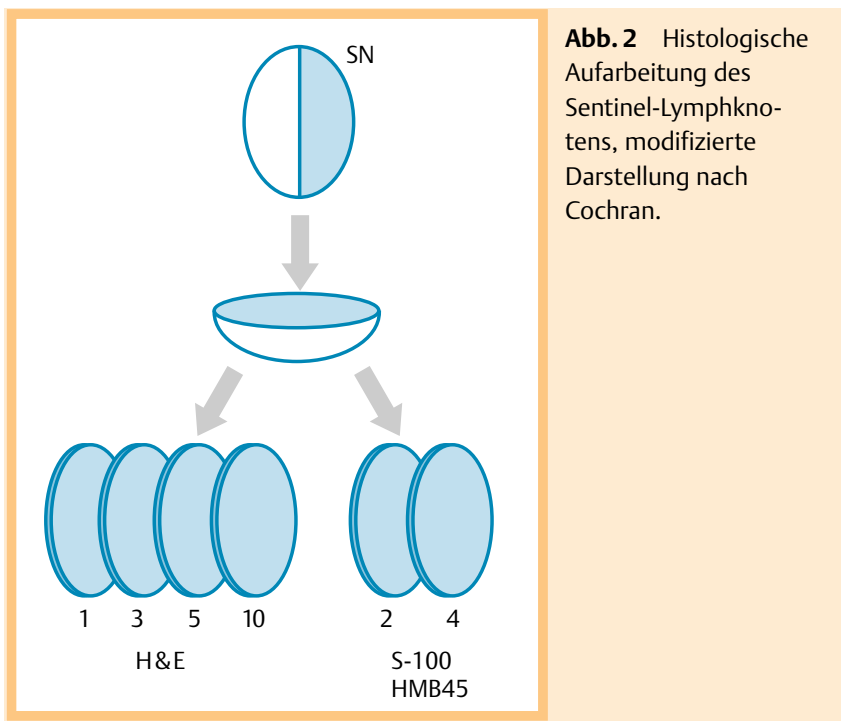

wendung [9] ( Abb. 2) [10]. Dieses Protokoll wurde wegen seiner relativ niedrigen Sensitivität (Detektionsraten im Durchschnitt etwa $18 \%$ ) kritisiert [11].

Eine alternative Möglichkeit der histologischen Aufarbeitung des SLK bietet die von Starz et al. empfohlene Vorgehensweise [12], die den Fokus beim makroskopischen Zuschnitt nicht auf die Medianebene des Lymphknotens legt und in approximativ 1-2 mm dicke Gewebescheiben parallel zur Längsachse aufgeschnitten wird. Dieses Vorgehen gewährleistet eine bessere Beurteilung der außerhalb der Mittelebenen gelegenen LK-Anteile und gewährleistet somit die Diagnose zusätzlicher, nicht in der mittleren Schnittebene sichtbarer Metastasen [12-14]. Starz et al. haben eine Detektionsrate von 27,5\% beschrieben. Mit einem ähnlichen Protokoll konnten wir eine Mikrometastasen-Detektionsrate in der gleichen Größenordnung erzielen [15].

Basierend auf dem Konzept der Halbierung des Lymphknotens und paramedianer Aufarbeitung haben Cook et al. das gegenwärtige EORTC-Protokoll entwickelt, bei dem je 20 Mikrotomschnitte jeder LK-Hälfte untersucht werden. Um tiefer in den SLN einzudringen, wurden in bestimmten Abständen („gaps“) von $50 \mu \mathrm{m}$ zwischen die zu färbenden Schnitte eingefügt, sodass letztlich eine maximal $0,8 \mathrm{~mm}$ breite Scheibe aus Lymphgewebe der Mittelebene relativ intensiv durchgemustert wird. Für dieses
Protokoll wurden Mikrometastasen-Detektionsraten von 33,8\% $[2,11]$ berichtet. Ein Nachteil dieser Vorgehensweise ist allerdings, dass die nachträgliche Beurteilung fragwürdiger Zellen die Anfertigung und Lagerung von Leerschnitten erforderlich macht.

In Göttingen kommen gewöhnlich 1 - 3 SLKs pro Patient zur Aufarbeitung (Median 2, Mittelwert 1,8 $\pm 0,9$ ). Wir haben ein den EORTC-Richtlinen entsprechendes, aber leicht modifiziertes Sentinel-Protokoll eingeführt, bei dem der Zuschnitt der gleichen ebenfalls mit der Halbierung des SLNs entlang der Längsachse begonnen wurde. Von jeder LK-Hälfte wurden 5 Serien von Paraffinschnitten von der Oberfläche beider Hälften angefertigt, mit H\&E gefärbt und mit immunhistochemischen Antikörpern (S100, MART-1, HMB45, Pan Melanoma plus) inkubiert und auch jeweils entsprechende Leerräume („gaps“) zwischen den einzelnen Serien eingehalten. Im Vergleich zum OriginalEORTC Protokoll wurde im Göttinger Protokoll zusätzlich der Antikörper MART-1 eingesetzt und die Anzahl der mit HMB45 inkubierten Schnitte erhöht. Um eine Eingrenzung des mit der Durchsicht der Objektträger verbundenen Arbeitsaufwands und der Kosten zu gewährleisten, wurden jeweils nur 5 Serien (anstatt 6) von Schnitten zur Auswertung angefertigt. Mit diesem, auf 42 Objektträger pro Patient begrenzten Sentinel-Protokoll wurde bei einer Anzahl von 128 SLNs eine Detektionsrate von $27 \%$ erreicht.

\section{Wertigkeit der immunhistochemischen Färbungen $\nabla$}

Im Gegensatz zu nodalen Metastasen von anderen malignen Tumoren sind Melanomzellen im lymphatischen Gewebe häufig in kleinen Gruppen, aber auch als Einzelzellen anzutreffen, die meist nur durch den Einsatz immunnhistochemischer Antikörper $(\mathrm{AK})$ entdeckt werden und deren Einführung die Detektionsraten um mindestens $10 \%$ gesteigert haben dürfte [16]. Der S-100 Antikörper weist eine hohe Sensitivität beim Auffinden von Melanomzellen im Lymphknotengewebe auf, färbt jedoch auch dendritische Zellen an, die in kleinen Haufen gelagert, von Melanozyten abgegrenzt werden müssen. Zum Nachweis von Einzelzellen und kleinen Zellhaufen hat sich in unserem Labor der Einsatz des Antikörpers MART-1 bewährt, der unserer Erfahrung nach eine etwas geringere Sensitivität als der AK gegen das S-100 Protein aufweist, im SLK aber spezifischer zur Detektion von Melanomzellen ist. HMB45 hat eine Sensitivität von nur etwa $70 \%$, kann aber bei der Diagnose von intranodalen Nävi hilfreich sein, deren Zellen für gewöhnlich HMB45 negativ sind, bzw. nur eine äußerst schwache zytoplasmatische Positivität zeigen. Nävuszellen finden sich in bis zu $14 \%$ der SLKs [17] und sind meist durch ihre Lokalisation in der fibrösen Kapsel und/ oder bindegewebigen Trabekel erkennbar. Die größte Herausforderung für den Befunder ist bei ausschließlich HMB45 zytoplasmatisch positiven Einzelzellen gegeben. Diese sind zytomorphologisch auf immunhistochemischen Schnitten nur eingeschränkt von Melanophagen zu differenzieren und auf nachfolgenden oder vorangehenden Schnittstufen häufig nicht (mehr) erfasst. Bei der von uns verwendeten Kombination an Antikörpern hat der AK Pan Melanoma plus keinen diagnostischen Zugewinn gezeigt. 


\section{Mikroanatomische Lokalisation der Metastasen im SLK}

Verschiedene Arbeitsgruppen haben versucht, unterschiedliche Kriterien der Metastasierung und ihre Ausbreitung im Lymphknoten herauszustellen um prognostische Faktoren herauszuarbeiten. In der auf Starz zurückgehenden S-Klassifikation wird die Eindringtiefe der Melanomzellen von der Innenseite der Kapsel, analog dem Breslow-Index beim Primärtumor, bestimmt [12]. Diese Einteilung (SI-SIII) erlaubt eine Voraussage bezüglich weiterer befallener Lymphknoten der gleichen Region und korreliert mit der des Gesamt-Überlebens. Dewar et al. [18] konnten einen klaren Zusammenhang zwischen der mikroanatomischen Lokalisation der Metastasen im SLK und dem Auffinden von zusätzlichen Metastasen bei der nachfolgenden kompletten Lymphknotendissektion (CLND) aufzeigen. Bei Patienten mit rein subkapsulärer Lokalisation der Metastasen konnten keine weiteren LKMetastasen in der CLND gefunden werden. In einer neueren Arbeit von Akkooi et al. [19] konnten bei Metastasen mit einer Ausdehnung $<0,1 \mathrm{~mm}$ im SLK keine zusätzlichen Absiedlungen in nachgeschalteten Non-Sentinel-Lymphknoten detektiert werden. Dieser Sachverhalt wirft verständlicherweise die Frage der Notwendigkeit einer CLND bei Patienten mit ausschließlich subkapsulärer Lokalisation, bzw. Eindringtiefe $<0,1 \mathrm{~mm}$ der Metastasen im SLK auf. Die Rolle von Einzelzellen [20], die sich mitunter nur schwer von normalerweise ortsständigen Zellen unterscheiden lassen, bleibt unklar und erfordert wohl sehr lange Nachbeobachtungszeiten.

\section{Status quo}

Die subtile pathologische Aufarbeitung der SLNs ist kostspielig und verlangt ausreichende Erfahrung vom Befunder. Sie ist in Zeiten zurückgehender Ressourcen nur schwer mit in den Routinebetrieb eines pathologischen Labors integrierbar. Die Frage der prognostischen Aussagekraft von bestimmten Metastasierungsmustern im SLK verlangt die Korrelation zur komplettierenden regionalen LK-Dissektion (CLND) und zu den klinischen Verläufen. Die prognostische Bedeutung von einer minimalen Tumorlast im SLK lässt sich wahrscheinlich nur in multizentrischen Studien mit Langzeitverläufen beurteilen.

\section{Abstract}

\section{Our Approach in Goettingen to Pathology of Sentinel Lymph Nodes for Melanoma}

$\nabla$

The pathological assessment of sentinel lymph nodes for melanoma contents a compromise between efficacy and expenditure of work, reflecting the existence of different protocols. The challenge for the pathologist is the detection of intranodal micrometastases and single cells by using a cocktail of antibodies. The prognostically impact of nodal metastatic patterns and minimal tumorload will have to be proved in multicentre trials.

\section{Literatur}

1 Kretschmer L, Beckmann I, Thoms KM et al. Sentinel lymphnodectomy does not increase the risk of loco-regional cutaneous metastases of malignant melanomas. Eur J Cancer 2005; 41: 531 - 538

2 van Akkooi ACJ, de Wilt JHW, Verhoef $C$ et al. High positive sentinel node identification rate by EORTC melanoma group protocol. Prognostic indicators of metastatic patterns after sentinel node biopsy in melanoma. EJC 2006; 42: 372 - 380

3 Abrahamsen HN, Hamilton-Dutoit SJ, Larsen J, Steiniche T. Sentinel lymph nodes in malignant melanoma. Extended histopathologic evaluation improves diagnostic precision. Cancer 2004; 100: 1683- 1691

4 Carlson GW, Murray DR, Lyles RH et al. The amount of metastatic melanoma in a sentinel lymph node: does it have prognostic significance? Ann Surg Oncol 2003; 10: 575-581

5 Estourgie SH, Nieweg OE, Valdés Olmos RA et al. Review and evaluation of sentinel node procedures in 250 melanoma patients with a median follow up of 6 years. Ann Surg Oncol 2003; 10: 681-688

6 Gershenwald JE, Thompson W, Mansfiled PF et al. Multi-institutional melanoma lymphatic mapping experience: The prognostic value of sentinel lymph node status in 612 stage I or II melanoma patients. J Clin Oncol 1999; 17: 976-983

7 Vuylsteke RJ, van Leeuwen PA, Statius Muller MG et al. Clinical outcome of stage I/II melanoma patients after selective sentinel lymph node dissection: long-term follow-up results. J Clin Oncol 2003; 21: 1057 1065

8 Cascinelli N, Belli F, Santinami $M$ et al. Sentinel lymph node biopsy in cutaneous melanoma: the WHO Melanoma Program experience. Ann Surg Oncol 2000; 7: 469-474

9 Cochran AJ, Wen DR, Morton DL. Occult tumor cells in the lymph nodes of patients with pathalogical stage I malignant melanoma: an immunohistological study. Am J Surg Pathol 1988; 12: 612-618

10 Cochran AJ. Surgical pathology remains pivotal in the evaluation of 'sentinel' lymph nodes. Am J Surg Pathol 1999; 23: 169- 1172

11 Cook MG, Green MA, Anderson B et al. The development of optimal pathological assessment of sentinel lymph nodes for melanoma. J Pathol 2003; 200: $314-319$

12 Starz H, Balda BR, Krämer KU et al. A micromorphometry-based concept for routine classification of sentinel lymph node metastases and its clinical relevance for patients with melanoma. Cancer 2001; 91: $2110-2121$

13 Bieligk SC, Ghossein R, Bhattacharya S, Coit DG. Detection of tyrosinase mRNA by reverse transcription-polymerase chain reaction in melanoma sentinel nodes. Ann Surg Oncol 1999; 6: 232 - 240

14 Ruiter DJ, Spatz A, van den Oord JJ, Cook MG, Pathology Committee of the European Organization Research and Treatment of Cancer (EORTC) Melanoma Group. Pathologic staging of melanoma. Semin Oncol 2002; 29: $370-381$

15 Kretschmer L, Bertsch HP, Meller J. Sentinel lymph node biopsy in malignant melanoma-an update. J Dtsch Dermatol Ges 2003; 1: 777 784

16 Messina JL, Glass LF, Cruse CW et al. Pathologic examination of the sentinel lymph node in malignant melanoma. Am J Surg Pathol 1999; 23: 686-690

17 Cook M, di Palma S. Our approach to pathology of sentinel lymph nodes for melanoma. J Clin Pathol 2008; 61: 897-902

18 Dewar DJ, Newell B, Green MA et al. The microanatomic location of metastatic melanoma in sentinel lymph nodes predicts nonsentinel lymph node involvement. J Clin Oncol 2004; 22: 3345-3349

19 van Akkooi ACJ, de Wilt JHW, Verhoef C et al. Clinical relevance of melanoma micrometastases $(<0.1 \mathrm{~mm})$ in sentinel nodes: are these nodes to be considered negative? Ann Oncol 2006; 17: 1578-1585

20 Satzger I, Völker B, Meier A et al. Prognostic significance of isolated HMB45 or Melan A positive cells in Melanoma sentinel lymph nodes. Am J Surg Pathol 2007; 31: 1175 - 1180 